\title{
Path Planning for Two-Link Navigation in an Unknown Environment using webcam
}

\author{
Shahed Shojaeipour ${ }^{1, a}$, Ali Khaki Sedigh ${ }^{2, b}$, Ali Shojaeipour ${ }^{3, c}$ \\ Edmund Ng Giap Weng ${ }^{4, d}$, Nooshin Hadavi $i^{3, e}$ \\ ${ }^{1}$ Dept. of Mechanical \& Material Eng. Universiti Kebangsaan Malaysia, Bangi, 43600, Malaysia \\ ${ }^{2}$ Faculty of Electrical Engineering, K.N. Toosi University of Technology, Tehran, Iran \\ ${ }^{3}$ Islamic Azad University - Shirvan Branch, Shirvan, Iran \\ ${ }^{4}$ Centre of Excellence for Semantic Technology and Augmented Reality CoESTAR \\ Universiti Malaysia Sarawak, 94300, Sarawak, Malaysia \\ aashojaeipour@gmail.com, bsedigh@kntu.ac.ir, cali.shojaeipour@gmail.com, dgwng@fcs.unimas.my, \\ enooshin.hadavi@gmail.com
}

Keywords: Voronoi Diagram, Image Processing, Vision Servo, Unknown Environment, Two-Link Manipulator.

\begin{abstract}
In this article, we used image processing by a webcam connected on top of the arm robot. The robot navigation is in an unknown environment. Then start point and target point were determined for the robot, so the robot needs to have a program for path planning using Voronoi diagrams to find the path. After the possible path for moving the robot was found, the route information obtained was sent to the arm robot. The arm robot moves in the workspace and any time new information was processed via the webcam. The program was written using MATLAB software which at controls the robot's movement the unknown environment.
\end{abstract}

\section{Introduction}

After returned the manuscript must be appropriately modified. Today, vision based sensors such as webcams are falling in price more rapidly than any other sensors. This type of sensor is also a richer sensor than traditional ranging device, more data simultaneously [1]. Consequently, visual servo control of robotic manipulators has become an area of rapid research and development over the last two decades.

Visual servo is the use of image data for manipulation and control of robot movement. Typically, the image of the robot workspace is captured, from which a target is identified. The position of the target is then estimated, and the corresponding robot joint angles and velocities are determined to enable the robot to reach its target.

In this work, we present the development of a visual servo which enables a two-link planar robotic manipulator to navigate itself though arbitrarily positioned obstacles. The image of the workspace plane is captured using a webcam. The image is then processed to identify the edges of objects within the workspace. A Voronoi diagram $(\operatorname{VD}(\mathrm{S}))$ is then constructed, marking paths that avoid these objects. The optimal path is then computed, which would then be used as the robot trajectory.

\section{Processing Unknown Environment Strategy}

This strategy can efficiently use the available information and reduce the planning time. Navigation in an unknown environment is a more challenging topic. For example, unmanned machines with navigation ability in unknown environments could perform tasks in many dangerous places that humans would not wish to entry for safety. Navigation in an unknown environment means no 